\title{
A Review of Coronagraphic Observations of Shocks Driven by Coronal Mass Ejections
}

\author{
Angelos Vourlidas* and Veronica Ontiveros ${ }^{\dagger, * *}$ \\ ${ }^{*}$ Code 7663, Naval Research Laboratory, Washington, DC 20375, USA \\ ${ }^{\dagger}$ Instituto de Geofisica, Universidad Nacional Autonoma de Mexico, DF, 04510, MEXICO \\ ${ }^{* *}$ CEOSR, George Mason University, Fairfax VA, 22030, USA
}

\begin{abstract}
The existence of shocks driven by Coronal Mass Ejections (CMEs) has always been assumed based on the superalfvenic speeds for some of these events and on indirect evidence such as radio bursts and distant streamer deflections. However, the direct signature of the plasma enhancement at the shock front has escaped detection until recently. Since 2003, work on LASCO observations has shown that CME-driven shocks can be detected by white light coronagraph observations from a few solar radii to at least $20 \mathrm{R}_{\text {sun }}$. Shock properties, such as the density compression ratio and their direction can be extracted from the data. We review this work here and demonstrate how to recognize the various shock morphologies in the images. We also discuss how the two-viewpoint coronagraph observations from the STEREO mission allow the reconstruction of the 3D envelope of the shock revealing some interesting properties of the shocks (e.g., anisotropic expansion).
\end{abstract}

Keywords: Coronal Mass Ejections, Interplanetary shocks, Shocks in Plasma,Coronagraphs PACS: 96.60.Ph, 96.50.Fm, 52.35.Tc, 95.55.Fw

\section{THE LONG SEARCH FOR CME-DRIVEN SHOCKS}

Ever since the accumulation of the first statistics on the properties of CMEs in the 1970s [3], it was realized that many CMEs propagate at speeds in excess of $1000 \mathrm{~km} / \mathrm{s}$ at a few $\mathbf{R}_{\text {sun }}$ above the solar surface which are higher than the Alfvén speed at these heights $(\sim 800 \mathrm{~km} / \mathrm{s}$; [13]). Therefore, it is expected that CMEs drive shocks in the corona and that the density compression at the shock front could lead to detectable signatures in coronagraph images.

The search for such signatures started with the Skylab images and [4, 1] where the first ones to report the existence of a faint front ahead of the main CME interpreting it as evidence of a bow shock. These so-called 'forerunners' were further analyzed by [6] and [7]. However, [9] demonstrated that, in the higher quality Solwind images, these features were part of the transient itself and were not associated solely or even consistently with fast events. Others suggested that the bright loop-like front of some transients was the enhancement from a fast MHD shock [15, 23] but it was quickly pointed out by [20] that slow events also exhibit such loop-like fronts. The works by [20] and [9] casted doubt on whether the density enhancement from the shock could ever be detected directly in the images. Indeed, most published CME shock 'detections' relied on indirect evidence such as distance streamer deflections [2, 16, 21] or deductive reasoning (e.g., fast lateral expansion, no streamer motion before the CME) [22].

While these analyses strongly supported the existence of CME-driven shocks, they did not provide unambiguous detection of shock signatures in coronagraph images. This was 
not a satisfactory situation. CME-driven shocks are the main (if not the only) accelerators of relativistic particles, the so-called solar energetic particle (SEP) events, which have major space weather implications. Many studies have indicated that the acceleration of SEPs occurs between 4-10 $\mathrm{R}_{\text {sun }}$ when the CME shock is at the coronagraph field of view. Furthermore, CME shocks are the main means of interaction between successive CMEs and may increase the geoeffectiveness of such events [11]. It is, therefore, important to be able to follow their evolution in the inner corona and compare observations with the sophisticated simulations currently available [10] .It is obvious that from a space weather and SEP analysis standpoints the direct detection and measurement of the parameters of CME driven shocks is critical. It is fortunate that the recent years has brought major advances in both of these issues.

The first direct detection of the density enhancement from a CME-driven shock was reported by [25] based on calibrated LASCO images. The authors used an MHD model of the event to verify that a shock was indeed expected at the observed location. They also provided the first direct connection between a shock and the associated streamer deflection and presented a few more examples of such shocks. This enabled [27] to recognize the density compression from the shock at the CME flanks and associate it with a type-III burst. More recently, [17] showed that faint fronts can be detected ahead of the majority of fast CMEs, they derived the density compression ratio at the shock front and demonstrated that the observed density profiles are consistent with line-ofsight (LOS) integration through a bowshock-like structure. For a few cases, [17] were able to estimate the direction of propagation of the shock.

This paper provides us with an opportunity to review the status of CME-driven shocks in coronagraph images, and explain how these shocks can be recognized in the observations and what parameters can be safely extracted from calibrated images. We also present preliminary observations of waves from the STEREO mission that can provide 3D information about the shape, direction of the shock and better estimates of the density compression ratio.

\section{WHERE ARE THE SHOCKS IN CORONAGRAPH IMAGES?}

Our experience with coronagraph image analysis suggests that the lack of unambiguous shock detections in the past is likely a result of the lower contrast, smaller field of view, and reduced image cadence of previous coronagraphs. The use of CCD imagers in the LASCO instruments and the nearly continuous solar monitoring over $30 \mathrm{R}_{\text {sun }}$ enable the detection of much fainter and finer structures in the images and allows a better understanding of the observed scene; namely, the decoupling of CME-related from other coronal structures.

As we will argue, shock (and more generally wave)-induced density enhancements are rather ubiquitous in the data but it is the use of calibrated images and a familiarity with the morphology of these shock signatures that allows an observer to distinguish them over the plethora of ejecta and streamer material that may lie along a given LOS. We demonstrate this via two, rather typical, shock morphologies that we have been able to identify in the LASCO data. 

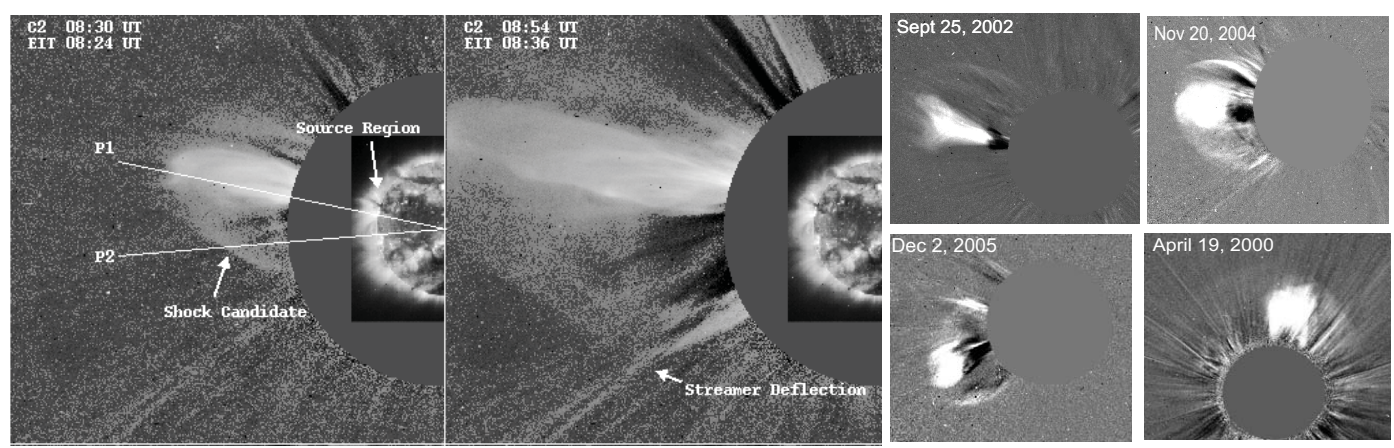

FIGURE 1. Examples of white light shock signatures with the bowshock morphology in LASCO images. Left panels: The April 2, 1999 event from Vourlidas et al (2003) showing the shock and streamer deflection. The lines labeled P1 and P2 mark the locations where density profiles were compared between observations and models. Right panels: Four more examples of bowshock-like events.

\section{Bowshock Morphology}

In this case, the images show a density enhancement propagating from the CME front along the CME flanks in a more or less straight line. It looks very similar to the waves emanating for the bow of a ship or of drawings of the bowshock of the terrestrial magnetosphere. The obvious similarities to fluid shocks made these features prime candidates for shock analysis and unsurprisingly were the first ones to be verified as shocks [25]. The two right panels in Fig. 1] shows a very good example of bowshock morphology taken from [25]. The shock is driven by a narrow ejection associated with a surge in the low corona. From our rather limited search in the LASCO database, it appears that this type of shock morphology is associated almost exclusively with narrow CMEs as can been seen in the other examples (four right panels, Fig. 11).

\section{"Double Front" Morphology}

CME-driven shocks can be recognized in the images by another characteristic morphology; a bright sharp loop-like feature proceeded by a much fainter front. Faint emission fills in the space between the two fronts. We use the term "double front" as a shorthand for referring to this morphology. The fainter front is usually difficult to detect in direct or even running difference images without some contrast enhancement procedure. These features are easily detected in calibrated images when a preevent image is subtracted. Examples of "double front" events are shown in Fig. 2. This morphology can be easily understood when one realizes that most CMEs are results of fluxrope eruptions [24]. Then, the bright loop is coronal plasma piled up at the top of the erupting fluxrope and the fainter front is the shock driven by the fluxrope. The fainter emission in-between the two front is just a result of integrating through the larger shock structure along the LOS. The faint front has been verified as a shock for one of the events (October 28, 2003) by [14] using an approach similar to [25]. We are very confident that this morphology is a robust indicator of a shock. Such morphologies have been seen in 3D MHD 

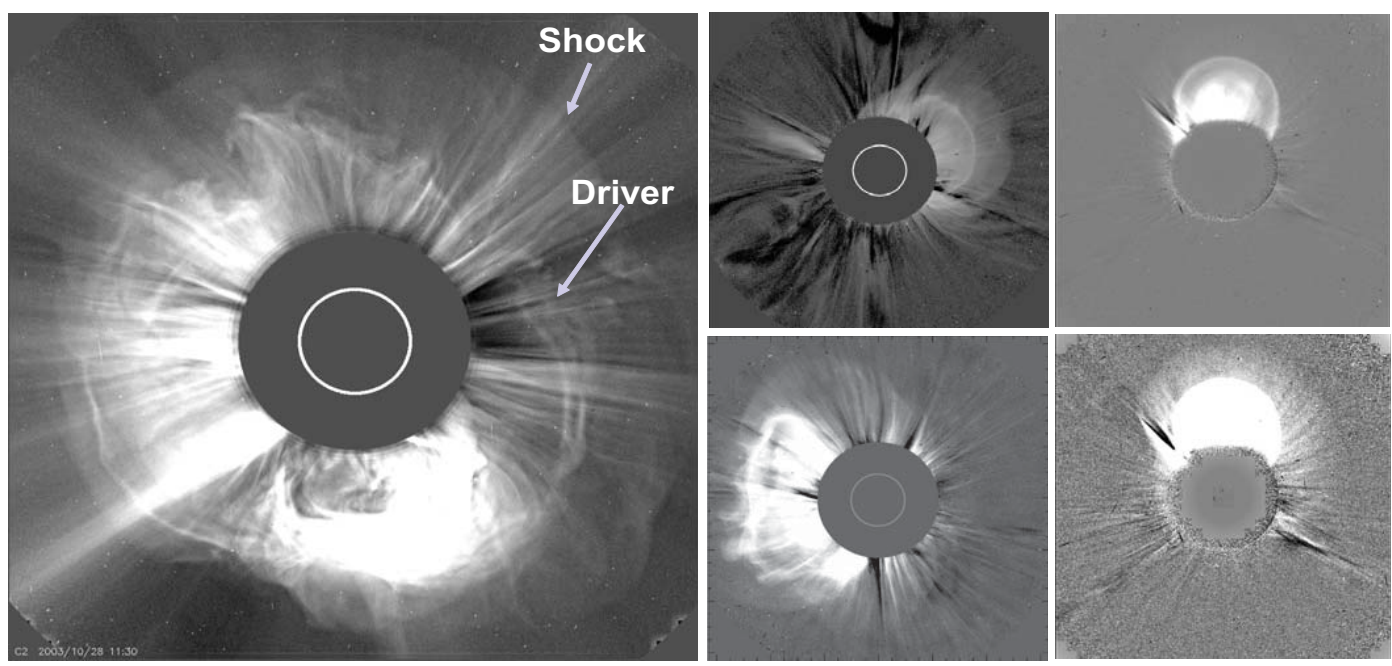

FIGURE 2. Examples of white light shocks with the 'double front' morphology in LASCO images. Right panel: The October 28, 2003 halo CME showing the shock and driver. Middle panels: Other examples of the "double front" morphology. Right panels: The same event can appear as a normal 3-part CME (top) or a "double front" (bottom) depending on the contrast (see text for discussion).

models [12] but have not been recognized in the images until now. This morphology is clearly associated with wide (partial or full halo) and fast CMEs, and with so-called 3-part structure CMEs. It appears then that the latter nomenclature is clearly a misnomer resulting from lower contrast of past observations (right panels of Fig. 2). The implications of this discovery and a detailed study of these events with comparisons to models will be discussed in an upcoming paper [26].

\section{EXTRACTION OF THE SHOCK PHYSICAL PARAMETERS}

The above morphologies are just two of the most easily recognized signatures of shock in the images. Shock fronts exhibit much more variability in the images. They can be continuous or appear over a small range of position angles. Sometimes they are detected ahead of the CME nose and sometimes not. Many times, no such fronts are seen ahead of fast CMEs but they can been seen in their flanks. Other times there is no evidence for a faint front anywhere around the image. In the majority of these cases, though, the CMEs propagate in the wake of another CME which disturbs the environment. Generally speaking almost all fast CMEs exhibit a faint front somewhere ahead of the main event. When such front is detected, we can extract some physical parameters from it, such as density compression ratio, speed, and even direction, as was shown by [17] (Fig. 3).

\section{Compression Ratios}

To derive a density compression ratio, we first estimate the density at the shock and the preevent density. The latter is usually determined by the inversion of partial 

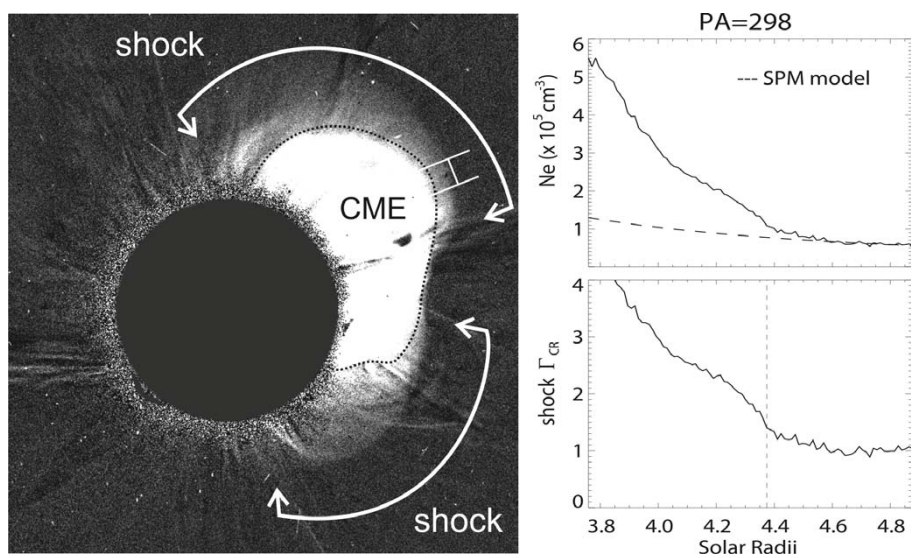

FIGURE 3. Density profile across the front of white light shocks. The shock, CME, and the location of the density profile are marked on the left panel. The excess density and compression ratio are shown on the right panel. The dashed line in the top panel is the assumed background density. The vertical dotted line in the bottom panel marks the location of the shock front.

polarized $(\mathrm{pB})$ images taken near the event. For the LASCO images, normally only one $\mathrm{pB}$ sequence is taken for each of the coronagraphs $(\mathrm{C} 2, \mathrm{C} 3)$. Alternatively, a standard density model such as the SPM [19] can be used. The shock density can be estimated by the measured excess mass (or number of electrons) at the shock front with the assumption of the (unknown) depth along the LOS. Results are shown in Fig. 3

For the events studied by [17], the resulting density jump is between 1.1 and 2.8 which is consistent with in-situ measurements and shows a reasonable correlation with the CME kinetic energy as expected (Fig. 4). Because the emission is optically thin, all white light measurements are subject to projection effects. The projection effects tend to confuse the shock with other intervening structures along the LOS (Fig 4) and smooth the density jump in the images compared to the sharp jumps observed with insitu instrumentation. [17] demonstrated that this is indeed the case by fitting the observed shock shape with a 3-dimensional geometric model of a bowshock shell with a width of $0.3 \mathrm{R}_{\text {sun }}$ and calculating the integrated emission from it. The resulting modeled density profiles fit very well the observed ones. This is encouraging and suggests that we may be able to extract 'true' density compression ratios from the images at heliocentric heights unreachable by in-situ probes but important for understanding particle acceleration.

\section{Shock Shape and Direction}

The geometric modeling of the shock provides another very important parameter: the direction of the shock nose. For the three events analyzed by [17] it was found that the shock nose was within $30^{\circ}$ of the radial from the likely source region. Although the reliability of this method needs to be validated with more events, it suggests that it could be possible to estimate the direction of a shock from single point coronagraph observations early in its evolution. 

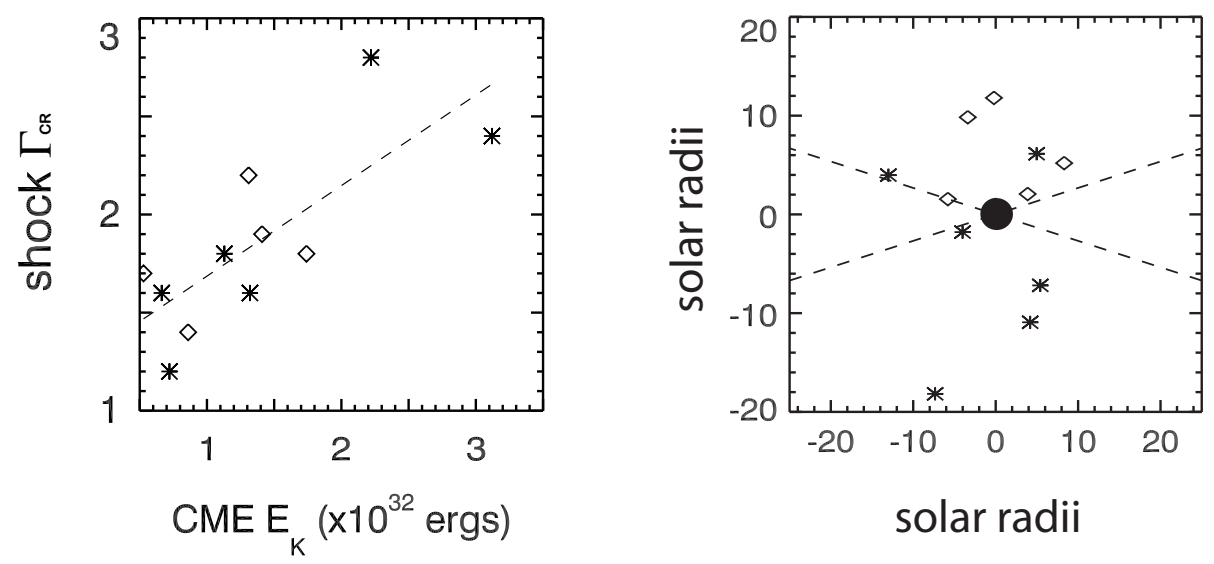

FIGURE 4. Analysis of shock parameters in [17]. Left panel: The density jump at the shock front shows a good correlation with the CME kinetic energy. Right panel: Plane of sky locations of the best shock signatures. The plot reveals that shock fronts are more visible when projected away from the streamer belt.

\section{3-Dimensional Shock Measurements}

Shock analysis will greatly benefit from the operation of the twin SECCHI coronagraphs suite [5] aboard the STEREO mission [8]. The ability to image the CME and its shock from two viewpoints simultaneously enables the fitting of the 3D shape of the shock with much more fidelity that it is possible from a single viewpoint. It also allows the easy separation of the shock from the driver and from other coronal features.

Because of the protracted solar activity minimum, there have been only a couple of shocks detected by SECCHI. Analysis of the December 31, 2007 event has shown that the shock direction and its propagation along the flanks of the streamer can be reconstructed in 3D [18]. Because of the higher sensitivity of the SECCHI coronagraphs it is now possible to reconstruct the waves driven by the slower CMEs which are common during solar minimum. Fig. 5 shows an example of simultaneous forward modeling of the CME-driven wave (light grey frame) and the CME itself (dark frame). The model shows that the CME propagates at $116^{\circ}$ west of the Sun-Earth line and the shock at $121^{\circ}$. It suggests that the shock and CME direction diverge. This is supported by observations at larger distances (not shown here). Another interesting result is the mismatch between the eastern extent of the model compared and the actual observations that show a much larger extent. It is obvious that a symmetric bowshock is not a good model for the actual shock which appears to propagate anisotropically in the corona. This behavior is expected since the morphology of the large scale coronal field will affect the propagation of an alfvenic wave by guiding it through the minimum of the local Alfvén speed. 

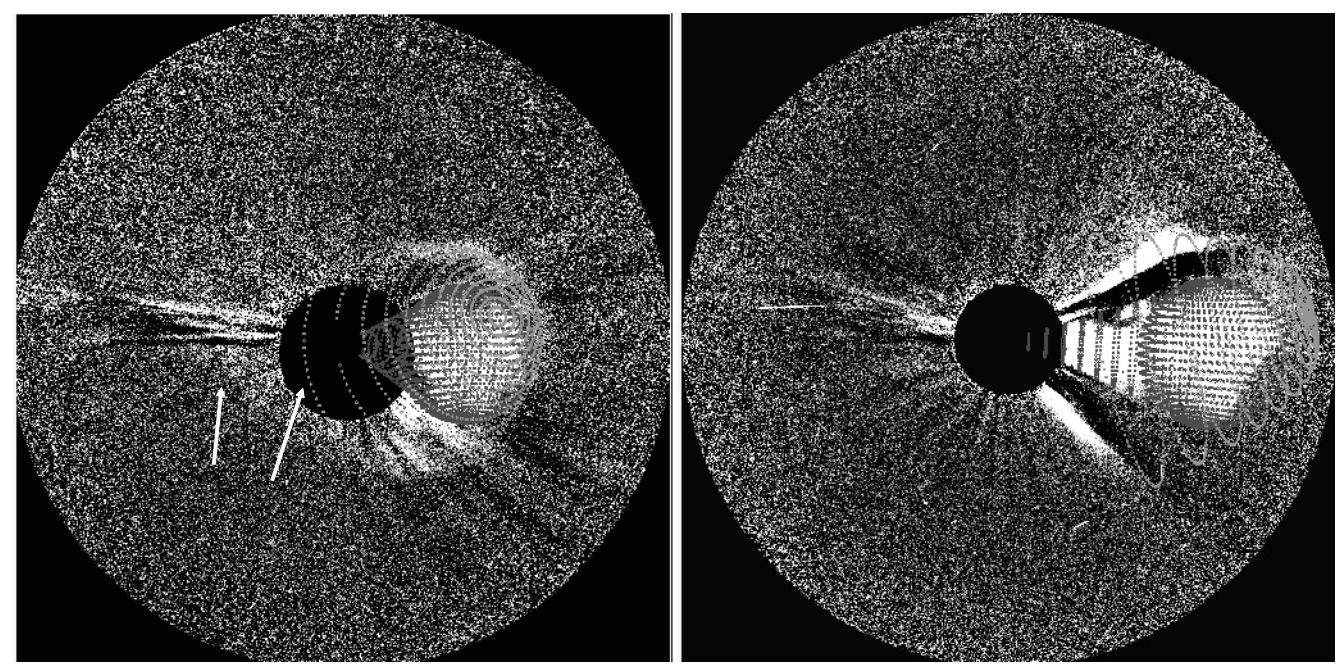

FIGURE 5. Example of the use of simultaneous SECCHI/COR2 observations for 3D forward modeling of a CME (dark frame) and its driven wave (light grey frame). The COR2-B (COR2-A) image is the left (right) panel. The arrows show that the eastern extent of the model does not agree with the observed wave and suggests that the shock does not propagate isotropically in the corona.

\section{CONCLUSIONS}

We cannot fully cover, in the small space allocated here, the implications from the recent advances in the detection and measurement of white light shocks. We give only a general overview of the results to demonstrate two things: (1) that CME-driven shocks can be easily detected in calibrated coronagraph images, and (2) that useful parameters of the shock properties can be extracted from these images. Plenty of work remains to be done but we have established some important facts:

- The observed shock intensities and shapes are consistent with an LOS integrates emission from a thin shell resembling a bowshock.

- The density compression ratios are less than 3, in agreement with in-situ measurements.

- The visibility of the shock depends strongly on the structures along the LOS. A preceding event may disturb the corona sufficiently to hamper detection of the faint shock signatures

- It is relatively straightforward to derive the direction of the shock from two viewpoint image. It may be possible to estimate the direction of the shock from single viewpoint images.

The ability to identify the shock structures, including the associated streamer deflections, is an important step towards the ultimate goal of the proper interpretation of coronagraph images. It will result in more accurate measurements of CMEs and their properties by enabling the observers to separate the ejecta from other structures in the images and by permitting a better connection with features seen in other regimes (e.g., EUV images). 
Finally, the analysis of white light shocks will greatly benefit the understanding of shock and particle acceleration physics when combined with off-limb spectroscopy and in-situ particle measurements like those planned for the upcoming Solar Orbiter mission.

\section{ACKNOWLEDGMENTS}

Part of this work was funded by the LWS TR\&T grant NNH06D85I. SOHO is an international collaboration between NASA and ESA. LASCO was constructed by a consortium of institutions: NRL (Washington, DC, USA), MPS (Katlenburg- Lindau, Germany), LAM (Marseille, France) and Univ.of Birmingham (Birmingham, UK). The SECCHI data are produced by an international consortium of the NRL, LMSAL and NASA GSFC (USA), RAL and Univ. Bham (UK), MPS (Germany), CSL (Belgium), IOTA and IAS (France).

\section{REFERENCES}

1. G. A. Dulk et al, Sol. Phys., 49, 369-394 (1976)

2. J. T. Gosling et al, J. Geophys. Res., 79, 4581-4587 (1974)

3. J. T. Gosling et al, Sol. Phys., 48, 389âĂŞ-397 (1976).

4. E. Hildner, et al, Sol. Phys., 42, 163-177 (1975)

5. R. A. Howard et al, Space Sci. Rev., 136, 67-115 (2008)

6. B. V. Jackson, and E. Hildner, Sol. Phys., 60, 155-170 (1978)

7. B. V. Jackson, Sol. Phys., 73, 133-144 (1981)

8. M. L. Kaiser et al, Sp. Sci. Rev, 136, 5-16 (2008)

9. J. T. Karpen, and R. A. Howard, J. Geophys. Res., 92, 7227-7234 (1987)

10. Y. C-M. Liu et al, Astrophys. J., 680, 757-763 (2008)

11. N. Lugaz, W. B. IV Manchester, and T. I. Gombosi, Astrophy. J., 634, 651-662 (2005)

12. B. J. Lynch et al, Astrophys. J., 617, 589-599 (2004)

13. G. Mann et al, "Coronal Transient Waves and Coronal Shock Waves", in Plasma Dynamics and Diagnostics in the Solar Transition Region and Corona, edited by A. Wilson, SOHO-8 Workshop Proceedings, ESA SP-446, Noordwijk, 1999, pp. 477-481

14. W. B. IV Manchester et al, Astroph. J., 684, 1448-1460 (2008)

15. A. Maxwell, and M. Dryer, Sol. Phys., 73, 313-329 (1981)

16. D. J. Michels et al., Adv. Sp. Res., 4, 311-321 (1984)

17. V. Ontiveros, and A. Vourlidas, Astrophy. J, 693, 267-275 (2009)

18. V. Ontiveros, A. Vourlidas, and P. Riley, SPD Mtg, June 14-18, 2009, Boulder, Abstract 22.12

19. K. Saito, A. I. Poland, and R. H. Munro, Sol. Phys., 55, 121-134 (1977)

20. D. G. Sime, M. R. MacQueen, and A. J. Hundhausen, J. Geophys. Res., 89, 2113-2121 (1984)

21. N. R. Sheeley, W. N. Hakala, and Y.-M. Wang, J. Geophys. Res., 105, 5081-5092 (2000)

22. D. G. Sime, and A. J. Hundhausen, J. Geophys. Res., 92, 1049-1055 (1987)

23. R. S. Steinolfson, "Theories of Shock Formation in the Solar Atmosphere", in Collisionless Shocks in the Heliosphere: Reviews of Current Research, edited by B. T. Tsurutani and R. G. Stone, Geophys. Monogr. Ser. 35, Washington, AGU, 1985, pp. 1-12

24. A. Thernisien, A. Vourlidas, R. .A Howard, Sol. Phys., 256, 111-130 (2009)

25. A. Vourlidas et al, Astroph. J., 598, 1392-1402 (2003)

26. A. Vourlidas et al, Astrophy. J., in preparation (2009).

27. Y. Yan et al, Sol. Phys., 239, 277-292 (2006) 